\title{
USO DE PRODUTOS NATURAIS COM FINS TERAPÊUTICOS EM PEDIATRIA
}

\author{
USE OF NATURAL PRODUCTS WITH THERAPEUTIC PURPOSES IN PEDIATRICS \\ USO DE PRODUCTOS NATURALES CON FINES TERAPÉUTICOS EN PEDIATRÍA
}

Luís Miguel Fernandes Nascimento (luis.miguel@ipb.pt) *

Maria Helena Pimentel (hpimentel@ipb.pt)**

Maria Ângela Gonçalves Rocha de Aragão (maria.aragao@ipb.pt) ***

\begin{abstract}
RESUMO
A literatura tem demonstrado que, no tratamento de doenças infantis, o uso de produtos naturais é particularmente adequado. Entendemos útil dispor de agentes terapêuticos cuja via de metabolização seja mais conhecida. $\mathrm{O}$ amplo índice terapêutico de muitos fitoterápicos e a sua tolerância criam boas perspetivas para a infância. Sendo considerada uma terapêutica segura, têm sido relatados efeitos adversos e interações decorrentes da administração simultânea de preparações à base de plantas e medicamentos convencionais (Izzo, 2005; Cunha, Ribeiro \& Roque, 2009). O presente estudo tem como objetivos conhecer a utilização de produtos naturais com fins terapêuticos em crianças e jovens, inscritos na consulta de pediatria da Unidade Local de Saúde do Nordeste (ULSN), e verificar se o recurso a esses produtos se deve ao aconselhamento de um profissional de saúde ou ao conhecimento empírico. Foram inquiridos todos os pais que acompanhavam os filhos na $1^{\mathrm{a}}$ consulta da especialidade, entre março e agosto de 2013, utilizando como instrumento de recolha de dados um questionário, elaborado par o efeito. Verificou-se que o uso de fitoterapia e de produtos naturais em pediatria não é muito frequente nos inquiridos em estudo. Nos casos em que ocorreu, a sua utilização fez-se por livre iniciativa e conhecimento empírico e, nem sempre, com indicações terapêuticas adequadas. Concluiuse pela necessidade de mais informação sobre esta temática, de forma a contribuir para uma mais efetiva, correta e responsável utilização destes produtos.

Palavras-chave: Produtos Naturais; Pediatria; Fitoterapia.
\end{abstract}

\begin{abstract}
The literature has been showing that the use of natural products is particularly suitable in the treatment of childhood diseases. It is extremely useful to have therapeutic agents whose metabolism pathway is better known. The wide therapeutic index of many herbal medicines and their tolerance create good perspectives to childhood. The herbal medicine is considered a safe therapy, but some adverse effects and interactions resulting from the concomitant use of herbal preparations and conventional drugs have been reported (Izzo, 2005; Cunha, Ribeiro \& Roque, 2009). The present study aims to know the use of natural products for therapeutic purposes in children and young people enrolled in the pediatric consultation of the Local Health Unit of the Northeast (ULSN) and verify if the use of these products
\end{abstract}




\section{Egitania \\ s c i e $\Omega$ c i a}

is a result of a health professional advice or empirical knowledge. From march to august 2013 all parents who accompanied their children to the 1st medical appointment were interviewed using a questionnaire drawn for data collection. It was verified that the use of phytotherapy and natural products in pediatrics is not very frequent in the study respondents. In cases where it occurred, its use was made by free initiative and empirical knowledge and not always with adequate therapeutic indications. It was concluded that there is a need for more information on this subject, in order to contribute to a more effective, correct and responsible use of these products.

Key words: Natural products; Pediatrics; Phytotherapy.

\section{RESUMEN}

La literatura demostró que en el tratamiento de enfermedades infantiles, el uso de productos naturales es particularmente adecuado. Entendemos útil disponer de agentes terapéuticos cuya vía de metabolización sea más conocida. El amplio índice terapéutico de muchos fitoterápicos y su tolerancia crean buenas perspectivas para la infancia. Si se considera una terapia segura, se han notificado efectos adversos e interacciones derivadas de la administración simultánea de preparados a base de plantas y medicinas convencionales (Izzo, 2005, Cunha, Ribeiro \& Roque, 2009). El presente estudio tiene como objetivos conocer la utilización de productos naturales con fines terapéuticos en niños y jóvenes inscritos en la cita de pediatría de la Unidad Local de Salud del Nordeste (ULSN) y verificar si el recurso a estos productos se debe al asesoramiento de profesional de salud o al conocimiento empírico. Se entrevistaron a todos los padres que acompañaban a los hijos en la primera consulta de la especialidad, de marzo a agosto de 2013, utilizando como instrumento de recogida de datos, un cuestionario, elaborado por el efecto. Se ha comprobado que el uso de fitoterapia y de productos naturales en pediatría no es muy frecuente en los encuestados estudiados. En los casos en que ocurrió, su utilización se hizo por libre iniciativa y conocimiento empírico y no siempre con indicaciones terapéuticas adecuadas. Se concluyó que era necesario más información sobre esta temática para contribuir a una más efectiva y correcta utilización.

Palabras clave: Produtos Naturales; Pediatria; Fitoterapia.

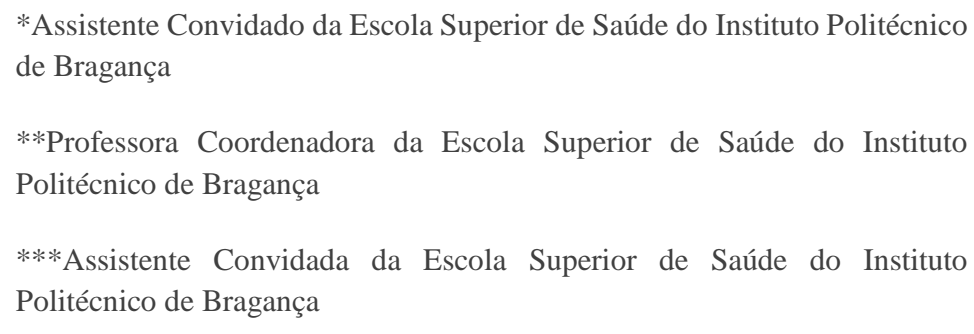

Submitted: 22th May 2017

Accepted: 04th December 2017 


\section{Egitania \\ $s$ c i e $\Omega$ c i a}

\section{INTRODUÇÃO}

O renovado interesse pela utilização das plantas medicinais originou uma crescente alteração das Farmacopeias (Steinhof, 2005). É o caso da presente Farmacopeia Portuguesa 9.0, adaptação e tradução da Farmacopeia Europeia 6 (Cunha \& Roque, 2011). Este interesse levou à criação de um grupo de trabalho permanente na Agência Europeia do Medicamento (AEM) constituído por peritos dos estados membros da União Europeia, do Parlamento Europeu, da Comissão Europeia e da Comissão da Farmacopeia Europeia (Steinhof, 2005).

A Organização Mundial de Saúde (OMS) define "fitoterapia ou fitomedicina a utilização de preparações fitoterápicas produzidas a partir dos materiais de origem vegetal desde a sua extração, fracionamento, purificação, concentração, ou outros processos físicos ou biológicos, podendo ser produzidos para consumo imediato ou como base para remédios e produtos herbáticos ou vegetais. Os produtos herbáticos podem conter excipientes ou ingredientes inertes, adicionados aos ingredientes ativos" (Organização Mundial de Saúde, 2001).

As plantas medicinais podem constituir uma alternativa segura e eficaz para o tratamento de distintas afeções, sendo cada vez maior o número de pessoas a recorrerem ao seu uso como complemento da medicação habitual (Garcia \& Solis, 2007; Martins, 2010). Podemos então definir fitoterapia como a ciência que estuda a utilização de produtos de origem vegetal com finalidades terapêuticas quer seja para prevenir, atenuar ou tratar um estado patológico (Garcia \& Solis, 2007; Fintelmann \& Weiss, 2010; Lopes, Oliveira, Lima, Formiga \& Freitas, 2013).

A terapia com medicamentos originados de plantas é relatada em sistemas de medicina milenares em todo o mundo, como a medicina chinesa, tibetana ou indiana (Fintelmann \& Weiss, 2010). Apesar de a farmacognosia ter surgido no século XVII, a área de estudo do uso de plantas medicinais e das suas aplicações para o tratamento de doenças a fitoterapia (do grego therapeia $=$ tratamento e phyton $=$ vegetal $)$ existe desde que se começou a compilar a informação acerca das plantas (Coelho, 2010). Na Europa, a fitoterapia surge como um sistema terapêutico separado baseado no uso tradicional de plantas e na extração de substância ativas das mesmas (Nascimento, 2013). Dentro da fitoterapia podemos reconhecer duas vertentes distintas a fitoterapia racional baseada em evidências científicas, como método de tratamento alopático, cuja eficácia dos produtos se encontra documentada em estudos farmacológicos e ensaios clínicos e a fitoterapia tradicional baseada na utilização de produtos derivados de plantas (Fintelmann \& Weiss, 2010; Bhattaram, Graefe, Kohlert, Veit \& Derendorf, 2002; Gruenwald, Brendler, Janicke, 2007).

As plantas sempre foram o principal meio a que a humanidade recorreu para o tratamento de doenças. Inicialmente de forma empírica (Knöss \& Chinou, 2012), posteriormente através da experimentação selecionando e conhecendo a atividade e toxicidade de muitas plantas, a maioria utilizadas na atualidade (Cunha, Silva, Roque \& Cunha, 2011).

A fitoterapia é somente uma parte do estudo mais abrangente das plantas medicinais, engloba a fitoquímica, a fitofarmácia, a farmacognosia e a fitofarmacologia (Fintelmann \& Weiss, 


\section{Egitania \\ $s$ c i e $\Omega$ c i a}

2010; Cunha, Silva, Roque \& Cunha, 2011). Para a compreensão da fitoterapia é indispensável conhecer a composição específica de substâncias que fazem parte de uma determinada planta. Atualmente é possível através de procedimentos físico-químicos analisar uma planta ou parte dela até ao mais ínfimo detalhe (Fintelmann \& Weiss, 2010). O estudo e aplicação de plantas medicinais ou de medicamentos fitoterápicos constituíram a base dos primeiros medicamentos. A maior parte dos medicamentos hoje obtidos por síntese química são extraídos de plantas (Murgia \& Pagiotti, 2011; Loureiro, 2012). O aparecimento de medicamentos obtidos de plantas por síntese química, veio diminuir o interesse do uso direto de plantas medicinais na terapêutica, em países desenvolvidos. Porém, na prescrição e utilização de medicamentos convencionais, por vezes não é relatado aos

Tabela 1 - Fármacos extraídos a partir de plantas (schulz, hansel \& tyler, 2002)

\begin{tabular}{cccc}
\hline Origem & Componente & Origem & Componente \\
\hline Beladona & Atropina & Efedra & Efedrina \\
Arbusto do café & Cafeína & Esporão do Centeio & Frgotamina \\
Arbusto do chá & Teofilina & Fava do Calabar & Cocaína \\
Casca do Teixo & Taxol & Folhas de coca & Pilocarpina \\
Casca da quina & Quinina & Folhas de Jaborandi & Emetina \\
Casca da quina & Quinidina & lpeca & Kava \\
Casca do Salgueiro & Salicina & Kawain & Morfina \\
Cólchico & Colquicina & Papoila do Ópio & Reserpina \\
Datura & Escopolamina & Rauvolfia & Penicilina \\
Dedaleira & Digoxina & Penicilium & \\
\hline
\end{tabular}

pacientes que a sua composição não é puramente química. Muitos dos medicamentos comercializados são naturais, mais propriamente de origem vegetal (Cunha, Roque \& Gaspar, 2011; Schulz, Hansel \& Tyler, 2002), como se pode observar na tabela 1.

A sua produção por síntese ou por processos biotecnológicos deve-se à sua perpetuação, ou seja, à manutenção através do espaço temporal das suas caraterísticas físicas e químicas, não havendo alteração de caraterísticas de ano para ano. Quanto às plantas medicinais a sua qualidade depende de vários fatores como o local e a forma como são colhidas, a estação do ano e a forma como são utilizadas frescas ou secas ou em extratos liofilizados (Cunha, Roque \& Gaspar, 2011). A investigação tem contribuído para racionalizar o seu uso de forma segura e eficaz, complementando o conhecimento.

Os portugueses como pioneiros dos descobrimentos tornaram as plantas acessíveis a todo o mundo em especial as oriundas da Índia, China, Brasil e África (Firmo, Meneses, Passos, Dias, Alves \& Dias, 2011). Em conformidade a medicina europeia deu um enorme salto qualitativo com os conhecimentos transmitidos pelos nossos navegadores e cientistas destacando-se Garcia de Orta, Tomé Pires e Amato Lusitano, entre outros. Em Portugal começaram a ser utilizadas de modo regular plantas até aí desconhecidas, afirmando assim a particularidade portuguesa neste processo. Por sua vez, proporcionaram ao espaço lusófono e intercontinental o conhecimento de plantas transportadas de uns locais para outros, tendose tornado numa placa giratória de divulgação deste conhecimento no mundo (Cunha, Ribeiro \& Roque, 2009; Cunha, Silva, Roque \& Cunha, 2011; Cunha, 2003; Cunha, Teixeira, Silva \& Roque, 2010). 


\section{Egitania \\ $\mathrm{s}$ c i e $\Omega$ c i a}

\section{ENTIDADES REGULADORAS DESTAS SUBSTÂNCIAS}

A AEM com sede em Londres é responsável pela avaliação científica dos medicamentos desenvolvidos pelas empresas farmacêuticas para uso no espaço europeu. Criada em 1995 presta assessoria aos estados-membros em questões de qualidade, eficácia e rigor no circuito do medicamento, apoia a indústria farmacêutica na investigação e procura harmonizar os requisitos de autorização a nível internacional (Gruenwald, Brendler \& Janicke, 2007).

Já o Grupo de Trabalho de Produtos à Base de Plantas Medicinais (GTPBPM) é um grupo de âmbito administrativo que trabalha sob supervisão da AEM e estabelece diretrizes para avaliar a eficácia, rigor e qualidade dos fitomedicamentos com base na literatura da Cooperativa Científica Europeia de Fitoterapia (CCEF) e da OMS. Publica dados que são postos à disposição das autoridades e requerentes nos processos de aprovação europeus descentralizados (Gruenwald, Brendler \& Janicke, 2007; Agência Europeia do Medicamento, 2013; Rägo \& Santoso, 2008) .

Também o Comité de Produtos Médicos à Base de Plantas (CPMBP) foi criado em setembro de 2004 pela AEM a partir do GTPBPM. Este comité fornece aos estados-membros da união europeia e suas instituições pareceres científicos relacionados com produtos medicinais à base de plantas. Um dos seus principais objetivos é elaborar literatura de forma a promover uma abordagem mais científica dos produtos derivados de plantas e uma lista de documentos relativos ao uso de substâncias e preparações à base de plantas (Agência Europeia do Medicamento, 2013).

Por sua vez a CCEF é uma organização fundada em 1989. Representa a fitoterapia nacional ou associações de medicamentos à base de plantas de toda a Europa e articula-se especialmente com Entidades Reguladoras de Medicamentos.

A Comissão E é um corpo científico criado pelo Instituto Federal das Drogas e Dispositivos Médicos Alemão, responsável pela validação da utilidade de centenas de fitoterápicos. Parte da informação usada pela Comissão E resulta de investimento privado (Schulz, Hansel \& Tyler,2002).

A Autoridade Nacional do Medicamento e Produtos de Saúde, I. P., abreviadamente designado por INFARMED, tem por missão regular e supervisionar o setor do medicamento, dispositivos médicos e produtos cosméticos e de higiene corporal, segundo os mais elevados padrões de proteção da saúde pública, garantir o acesso dos profissionais de saúde e dos cidadãos a medicamentos, dispositivos médicos, produtos cosméticos e de higiene corporal, de qualidade, eficazes e seguros. Presta e recebe colaboração dos serviços e organismos da administração direta e indireta, ou autónoma, do Estado no âmbito das suas atribuições ( INFARMED, 2013).

No que diz respeito à legislação o Decreto-Lei n. ${ }^{0}$ 176/2006, de 30 de agosto, estabelece o regime jurídico a que obedece a autorização de introdução no mercado e suas alterações, o fabrico, a importação, a exportação, a comercialização, a rotulagem e informação, a 


\section{Egitania \\ $s$ c i e $\Omega$ c i a}

publicidade, a farmacovigilância e a utilização de medicamentos para uso humano e respetiva inspeção, incluindo medicamentos homeopáticos, radiofarmacêuticos e medicamentos tradicionais à base de plantas passando a incluir a Diretiva 2004/24/CE do Parlamento Europeu e do Conselho, de 31 de março de 2004.

A Lei ${ }^{\circ}$ 71/2013 regulamenta a Lei . $^{\circ}$ 45/2003, de 22 de agosto, relativamente ao exercício profissional das atividades de aplicação de terapêuticas não convencionais e regula o acesso às profissões no âmbito destas terapêuticas, nas quais se inclui a fitoterapia reconhecendo a autonomia técnica e deontológica do respectivo exercício profissional.

\section{FITOTERAPIA EM PEDIATRIA}

Nas últimas décadas, tem-se verificado um aumento da procura de tratamentos simples e naturais para prevenir ou tratar as patologias mais comuns da infância (Gruenwald, Brendler \& Janicke, 2007). Cada vez mais, os pediatras evitam prescrever medicamentos de síntese química de forma indiscriminada, devido aos reconhecidos efeitos adversos (Loureiro, 2012). Porém, a utilização de plantas com propriedades medicinais não deve nunca substituir por completo a terapêutica com medicamentos convencionais. As plantas medicinais quando usadas corretamente apresentam diversas vantagens comparativamente aos medicamentos de síntese química, tais como:

- Menor possibilidade de efeitos colaterais;

- Menor risco de toxicidade para o organismo;

- Não resistência a antibióticos;

- Produtos metabolizados e excretados não nocivos para o ambiente (Cunha, Ribeiro \& Roque, 2009; Cunha \& Roque, 2011; Loureiro,2012; Cunha, Teixeira, Silva \& Roque, 2010).

Segundo os mesmos autores, a utilização de medicamentos fitoterápicos ou mesmo de plantas medicinais é por vezes indicada pelo médico nomeadamente pelo pediatra, aquando das consultas de rotina ou mesmo em consultas de urgência pediátrica (Cunha, Ribeiro \& Roque, 2009; Cunha \& Roque, 2011; Loureiro,2012; Cunha, Teixeira, Silva \& Roque, 2010).

Muitas vezes os medicamentos utilizados no tratamento de patologias simples, nas cólicas por exemplo, são medicamentos à base de plantas como camomila, funcho, tília e cidreira. A sua utilização na forma de infusão é comum e nem sempre tem sido alvo da devida atenção, levando ao risco de utilização simultânea de medicamentos convencionais e plantas medicinais, não tendo em conta a possível interação entre ambos, potencialidade, sinergia, diminuição ou até inibição do efeito farmacológico do medicamento.

A utilização de um método pouco utilizado em Portugal, denominado de reconciliação terapêutica (Sanchez, López, Cresoimonjo \& Soler, 2008; Pires, 2011), permite aceder a todo o histórico farmacológico do doente, devendo para isso, o doente ou, neste caso concreto, os pais, fornecer elementos aquando de um episódio de urgência ou consulta de pediatria, para 


\section{Egitania \\ $s$ c i e $\Omega$ c i a}

que os profissionais de saúde tenham reunidos todos os dados no sentido de uma correta prescrição, sem riscos para a criança.

Os medicamentos fitoterápicos são usados nas causas da doença ou para mitigar os seus sintomas. A escolha do medicamento baseia-se no pressuposto de que os agentes ativos reparam as deficiências fisiológicas do corpo humano através de interações bioquímicas que se produzem nas células (Gruenwald, Brendler \& Janicke, 2007).

Os legisladores, em Portugal o INFARMED, aplica as mesmas diretrizes aos fitofármacos e aos medicamentos sintéticos. Ambos deverão ter um efeito farmacológico claramente demonstrado, assim como uma eficácia clínica.A sua segurança deve ser apoiada sempre por dados toxicológicos. O pedido de introdução de um novo medicamento deve ser efetuado pelo departamento competente, ou seja, por pessoal com conhecimentos na área da fitoterapia. A um produto com princípios ativos de eficácia comprovada aplica-se a regra comum a outros medicamentos (Gruenwald, Brendler \& Janicke, 2007).

Hoje em dia temos acesso a um arsenal terapêutico fitoterápico com inúmeras referências para diversas situações clínicas. Porém, tendo em conta o paciente, neste caso a criança, assim como acontece na medicina convencional e com os tratamentos farmacológicos, as plantas e os medicamentos utilizados, requerem uma utilização restrita e cuidadosa. Salientar que os estudos e ensaios clínicos de medicamentos praticamente não incluem crianças, gestantes e lactentes. A Academia Americana de Pediatria reconhece o uso crescente das medicinas complementares, nomeadamente da fitoterapia, em crianças e a necessidade de mais informação e apoio aos profissionais de saúde, sobretudo, aos pediatras (Kemper, Vohra \& Walls, 2008). Também se verifica idêntica realidade no Reino Unido e no mundo ocidental (Lorenc, Ilan-Clarke, Robinson \& Blair, 2009).

O amplo índice terapêutico de muitos fitoterápicos e a sua tolerância criam boas perspetivas para a infância. Nas doenças da infância procura-se estimular o sistema imunitário aumentando a resistência do organismo (Sibinga, Ottolini, Duggan \& Wilson, 2004; Mears, 2010). Este efeito obtém-se mais facilmente com fitoterápicos, do que com medicamentos de síntese (Gruenwald, Brendler \& Janicke, 2007). Os medicamentos convencionais têm como finalidade atuar em sintomas ou efeitos isolados, o que acarreta riscos de efeitos indesejados. O mesmo não acontece com os fitoterápicos, no entanto, o uso de fitoterápicos não deve ser abusivo e nalguns casos não recomendado em pediatria (Fintelmann \& Weiss, 2010; Sibinga, Ottolini, Duggan \& Wilson, 2004; Ceolin, Heck, Barbieri, Schwartz, Muniz \& Pillon, 2011).

A Academia Americana de Pediatria tem como objetivo otimizar o bem-estar das crianças e cuidados de saúde centralizados na família e como tal reconhece o uso crescente da medicina complementar e mais propriamente da fitoterapia (Kemper, Vohra \& Walls, 2008). Surge, assim, a necessidade de fornecer informações, apoio e aconselhamento farmacoterapêutico aos profissionais de saúde e aos pais, considerações éticas e análise custo-eficácia destes produtos, na opção pelo tratamento que melhor responda às necessidades de saúde da criança. 


\section{Egitania \\ $s$ c i e $\Omega$ c i a}

\section{OBJETIVOS}

Conhecer a utilização de produtos naturais com fins terapêuticos em crianças e jovens inscritos na consulta de pediatria da Unidade Local de Saúde do Nordeste (ULSN) e verificar se o recurso a estes produtos se deve ao aconselhamento de profissional de saúde ou ao conhecimento empírico.

\section{MATERIAL E MÉTODOS}

Estudo observacional e transversal. Foram considerados todos os pais (pai ou mãe) que acompanhavam os filhos na primeira consulta da especialidade de pediatria na ULS NordesteUH Bragança, no período de março a agosto de 2013. O número de consultas anuais desta especialidade ronda os 2500 , envolvendo 500 primeiras consultas. Os questionários foram aplicados pelos enfermeiros da consulta. Para acautelar a não ocorrência de repetição de participantes consideramos apenas as crianças e jovens que recorreram à primeira consulta, no período atrás descrito, até obter $30 \%$ de inquiridos em relação à população alvo. Aplicaram-se 120 questionários com validação de 114.

Aplicou-se um questionário estruturado de acordo com o estado da arte e evidência científica do tema em análise, baseado em autores da área (Murgia \& Pagiotti, 2011; Loureiro, 2012).

O pedido de aplicação do IRD foi submetido a autorização da Comissão de Ética da Unidade Local de Saúde do Nordeste (ULSN). O tratamento dos dados foi conseguido utilizando o programa estatístico S.P.S.S. 20.

\section{RESULTADOS}

Analisando a tabela 2 verifica-se que a idade dos pais é em média de 38,74 anos e das mães de 36,04. Quanto ao número de elementos do agregado familiar verificaram-se famílias com o número mínimo de 2 elementos. Enquadram-se nesta variável as famílias monoparentais. A média ronda os 4 elementos. Verifica-se ainda um número elevado de famílias apenas com um filho.

A idade das crianças e jovens em estudo tem um valor médio de 6 anos, com uma moda de 1 ano e um desvio padrão de 4,35. Os valores em falta, no caso da idade do pai, reportam-se a respostas em branco. 
Tabela 2 - Caraterização da população em estudo quanto à idade dos pais, $\mathrm{n}^{\circ}$ de elementos do agregado familiar e idade da criança/jovem.

\begin{tabular}{ccccc}
\hline & Idade Pai & Idade Mãe & $\mathbf{N}^{\circ}$ de elementos do agregado familiar & $\begin{array}{c}\text { Idade da criança/iovem } \\
\text { (anos) }\end{array}$ \\
\hline $\mathrm{N}$ & 111 & 114 & 114 & 114 \\
Média & 38,74 & 36,04 & 3,62 & 5,83 \\
Mediana & 39 & 36 & 4 & 5 \\
Moda & 40 & 36 & 4 & 1 \\
Desvio padrão & 6,945 & 6,529 & 0,775 & 4,35 \\
Mínimo & 21 & 19 & 2 & $<1$ \\
Máximo & 55 & 56 & 6 & 17 \\
\hline
\end{tabular}

No que diz respeito à área de residência 56,8\% residem em cidades, $17,8 \%$ em vilas e $25,4 \%$ têm residência oficial em aldeias (tabela 3). Quanto às habilitações académicas verifica-se uma maior percentagem de qualificações de ensino secundário ou equivalente no pai $(31,9 \%)$, seguidas do bacharelato/licenciatura $(21,6 \%)$. Na mãe, prevalece também o ensino secundário com 36,3\%. O bacharelato/licenciatura apresenta valores percentuais de 34,5\%. É de salientar, no entanto, um menor nível de escolaridade dos pais comparativamente às mães. Verifica-se ainda que cerca de $72 \%$ dos inquiridos possuem como habilitações académicas o ensino básico (41,4\% para os três ciclos), seguido do ensino secundário $(31,9 \%)$.

As profissões do pai são diversas $22,8 \%$ pertencem à classe de operários, artífices e trabalhadores similares e $21 \%$ exercem profissões do grupo de especialistas das profissões intelectuais e científicas. No caso da mãe este valor sobe para $28 \%$. A população não ativa assume nesta amostra um valor percentual reduzido.

No que se reporta à utilização de plantas medicinais obtiveram-se os resultados refletidos na tabela 4. Globalmente verifica-se que a grande maioria dos pais refere não recorrer ao uso destas substâncias no tratamento das patologias mais comuns da infância e em análise neste estudo. 
Tabela 3 - Caraterização académica e profissional dos pais

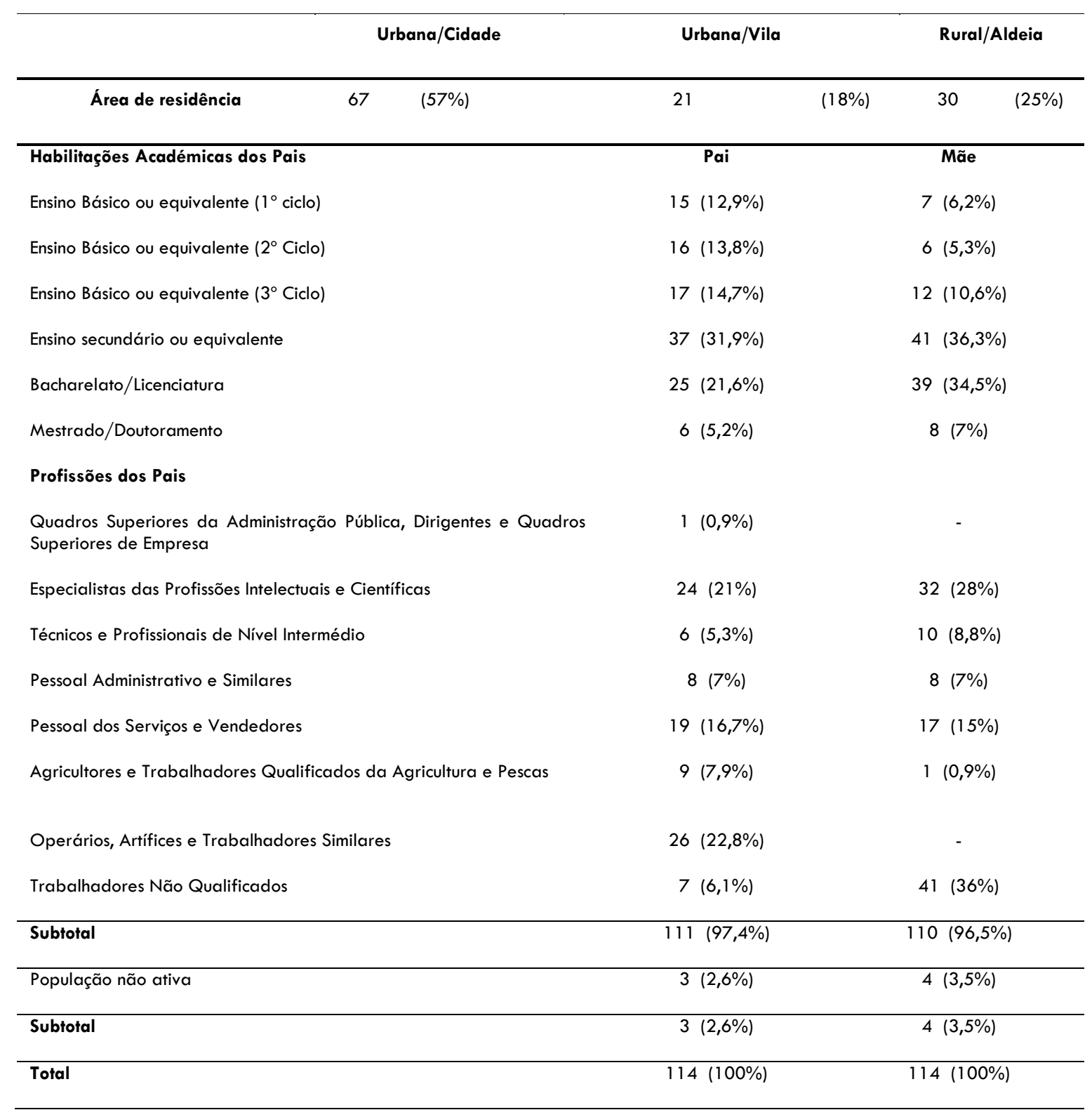

No que se reporta à utilização de plantas medicinais obtiveram-se os resultados refletidos na tabela 4. Globalmente verifica-se que a grande maioria dos pais refere não recorrer ao uso destas substâncias no tratamento das patologias mais comuns da infância e em análise neste estudo. 
Tabela 4 - Frequência de utilização, na população em estudo, de plantas/produtos naturais em afeções pediátricas

\begin{tabular}{|c|c|c|c|c|}
\hline & Sim, sempre & $\begin{array}{l}\text { Sim, com } \\
\text { bastante } \\
\text { frequência }\end{array}$ & $\begin{array}{l}\text { Sim, às } \\
\text { vezes }\end{array}$ & Não, nunca \\
\hline $\begin{array}{l}\text { Costuma recorrer à utilização de plantas } \\
\text { medicinais nas afeções respiratórias }\end{array}$ & $1(0,8 \%)$ & $8(6,8 \%)$ & $27(22,9 \%)$ & $80(67,8 \%)$ \\
\hline $\begin{array}{l}\text { Costuma recorrer à utilização de plantas } \\
\text { medicinais nas gripes e constipações }\end{array}$ & $2(1,7 \%)$ & $6(5,1 \%)$ & $45(38,1 \%)$ & $64(54,2 \%)$ \\
\hline $\begin{array}{l}\text { Costuma recorrer à utilização de plantas } \\
\text { medicinais na tosse }\end{array}$ & $2(1,7 \%)$ & $8(6,8 \%)$ & $44(37,3 \%)$ & $62(52,5 \%)$ \\
\hline $\begin{array}{l}\text { Costuma recorrer à utilização de plantas } \\
\text { medicinais nas afeções da orofaringe }\end{array}$ & $1(0,8 \%)$ & $3(2,5 \%)$ & $11(9,3 \%)$ & $102(86,4 \%)$ \\
\hline $\begin{array}{l}\text { Costuma recorrer à utilização de plantas } \\
\text { medicinais nas perturbações do sono }\end{array}$ & $3(2,5 \%)$ & $3(2,5 \%)$ & $24(20,3 \%)$ & $87(73,7 \%)$ \\
\hline $\begin{array}{l}\text { Costuma recorrer à utilização de plantas } \\
\text { medicinais na obstipação }\end{array}$ & $2(1,7 \%)$ & - & $17(14,4 \%)$ & $98(83,1 \%)$ \\
\hline $\begin{array}{l}\text { Costuma recorrer à utilização de plantas } \\
\text { medicinais nas cólicas }\end{array}$ & $1(0,8 \%)$ & $1(0,8 \%)$ & $24(20,3 \%)$ & $57(48,3 \%)$ \\
\hline $\begin{array}{l}\text { Costuma recorrer à utilização de plantas } \\
\text { medicinais na dermatite atópica }\end{array}$ & - & - & $6(5,1 \%)$ & $111(94,1 \%)$ \\
\hline
\end{tabular}

No caso da dermatite atópica $94,1 \%$, ou seja, a quase totalidade dos inquiridos não recorre a esta terapêutica, uma vez que apenas 6 pais assinalaram o recurso a plantas para tratar esta afeção dos filhos.

Também as afeções da orofaringe $(86,4 \%)$ e a obstipação $(83,1 \%)$ apresentam valores percentuais muito elevados de não utilização. O recurso a plantas é mais elevado nas gripes e constipações $(38,1 \%)$, tosse

$(37,3 \%)$ e afeções respiratórias $(22,9 \%)$, sobretudo na opção de resposta "sim às vezes". As perturbações do sono e as cólicas assumem valores de representação com alguma expressividade estatística (20,3\%), para ambas as situações.

Atendendo ao elevado número de plantas e outros produtos naturais (frutas, legumes e mel) utilizados, individualmente ou em conjunto, cuja resposta resultou de uma questão aberta, de forma a facilitar a leitura e a interpretação dos resultados agruparam-se as várias respostas para a mesma planta/produto, conforme tabela 5. Na questão referente às afeções respiratórias verificámos que o eucalipto recebe o maior número de respostas $(15,3 \%)$, em associação a outras plantas soma $18,5 \%$.

No caso das gripes e constipações o limão é o mais utilizado $(9,8 \%)$, seguido do eucalipto isolado ou em associação, totalizando 6,6\%. Para esta afeção o mel obtém 8,3\% de respostas. No caso da tosse constatámos que os inquiridos admitem que por vezes recorrem a substâncias naturais como o mel $(14,9 \%)$, o limão $(7,4 \%)$ e a cenoura $(9,1 \%)$ (sob a forma de xarope). Estas substâncias ao exercerem um efeito emoliente sobre as mucosas, por ação do açúcar, acalmam ou resolvem o problema da tosse. 


\section{Eqitania \\ s c i e $\Omega$ c i a}

Nos problemas de orofaringe verificámos uma maior utilização do limão $(1,6 \%)$, da tília $(0,8 \%)$, da sálvia $(0,8 \%)$, da cebola $(0,8 \%)$, do mel $(0,8 \%)$ e do poejo $(0,8 \%)$.

Nas perturbações do sono a camomila surge com maior percentagem de respostas $(13,5 \%)$, o que evidencia o conhecimento empírico e os benefícios da planta por parte dos utilizadores. Segue-se a tília com um total de $2,4 \%$ respostas.

Na obstipação os inquiridos referem uma menor utilização, no entanto, reconhecem no kiwi e na maçã reineta propriedades laxantes. O xarope de maçã reineta é mesmo o mais utilizado, observando-se uma percentagem de respostas que somadas totalizam 5,9\%.

Menor valor percentual assume o sene $(0,8 \%)$, a laranja $1,6 \%$, o funcho $2,5 \%$ e as sementes de linhaça $0,8 \%$. De referir que $0,8 \%$ de inquiridos recorrem a chás, não especificando as plantas usadas na infusão.

No caso das cólicas a maior utilização recai sobre a cidreira $(5,7 \%)$ e o funcho $(4,1 \%)$, mas também sobre a camomila com $3,4 \%$ de respostas. $\mathrm{Na}$

dermatite atópica apesar de 6 participantes assumirem o uso desta terapia, surge apenas 1 resposta $(0,8 \%)$ de identificação da substância e recai sobre a camomila.

Tabela 5 - Recurso a plantas/produtos naturais em afeções pediátricas por parte dos inquiridos

\begin{tabular}{|c|c|c|c|c|c|c|c|c|}
\hline & $\begin{array}{l}\text { Afeções } \\
\text { respiratóri } \\
\text { as }\end{array}$ & $\begin{array}{c}\text { Gripes e } \\
\text { constipaç } \\
\text { ões }\end{array}$ & Tosse & $\begin{array}{l}\text { Afeções } \\
\text { da } \\
\text { orofaring } \\
\text { e }\end{array}$ & $\begin{array}{c}\text { Perturbaç } \\
\text { ões do } \\
\text { sono }\end{array}$ & $\begin{array}{c}\text { Obstipaçã } \\
0\end{array}$ & Cólicas & $\begin{array}{c}\text { Dermatite } \\
\text { atópica }\end{array}$ \\
\hline Alecrim & $0,8 \%$ & - & - & - & - & - & - & - \\
\hline Camomila & $0,8 \%$ & $3,2 \%$ & $0,8 \%$ & - & $13,5 \%$ & - & $3,4 \%$ & $0,8 \%$ \\
\hline Carqueja & - & $0,8 \%$ & - & - & - & - & - & - \\
\hline Cebola & $0,8 \%$ & - & $3,5 \%$ & $0,8 \%$ & - & - & - & - \\
\hline Cenoura & - & $1,7 \%$ & $9,1 \%$ & - & - & - & - & - \\
\hline Cidreira & $2,4 \%$ & $1,7 \%$ & $2,4 \%$ & - & $0,8 \%$ & - & $5,7 \%$ & - \\
\hline Eucalipto & $18,5 \%$ & $6,6 \%$ & $1,7 \%$ & - & - & - & - & - \\
\hline Funcho & $0,8 \%$ & - & $1,7 \%$ & - & - & $2,5 \%$ & $4,1 \%$ & - \\
\hline Hortelã-pimenta & - & $1,7 \%$ & - & - & - & - & - & - \\
\hline Limão & $0,8 \%$ & $9,8 \%$ & $7,4 \%$ & $1,6 \%$ & - & - & - & - \\
\hline Limonete & $0,8 \%$ & $1,7 \%$ & - & - & - & - & - & - \\
\hline
\end{tabular}




\begin{tabular}{ccccccccc} 
Maçã-reineta & - & - & - & - & - & $5,9 \%$ & - & - \\
Malva & - & - & - & - & - & $0,8 \%$ & $0,8 \%$ & - \\
Mel & $1,6 \%$ & $8,3 \%$ & $14,9 \%$ & $0,8 \%$ & - & - & - & - \\
Mentas & $1,6 \%$ & $1,7 \%$ & - & - & - & - & - & - \\
Salvia & $1,6 \%$ & - & - & $0,8 \%$ & - & - & - & - \\
Sene & - & - & - & - & - & $0,8 \%)$ & - & - \\
Tilia & - & $1,7 \%$ & $0,8 \%$ & $0,8 \%$ & $2,4 \%$ & - & - & - \\
Outras & $4 \%$ & $4 \%$ & $4,8 \%$ & $0,8 \%$ & $0,8 \%$ & $3,4 \%$ & $1,7 \%$ & - \\
\hline
\end{tabular}

Quanto à forma de acesso a plantas/produtos naturais podemos observar no Gráfico 1 que $64 \%$ dos inquiridos referem utilizá-las por livre iniciativa, o que revela um conhecimento empírico das mesmas. Apenas $16 \%$ recorrem à sua utilização por indicação médica e $14 \%$ por aconselhamento farmacêutico.

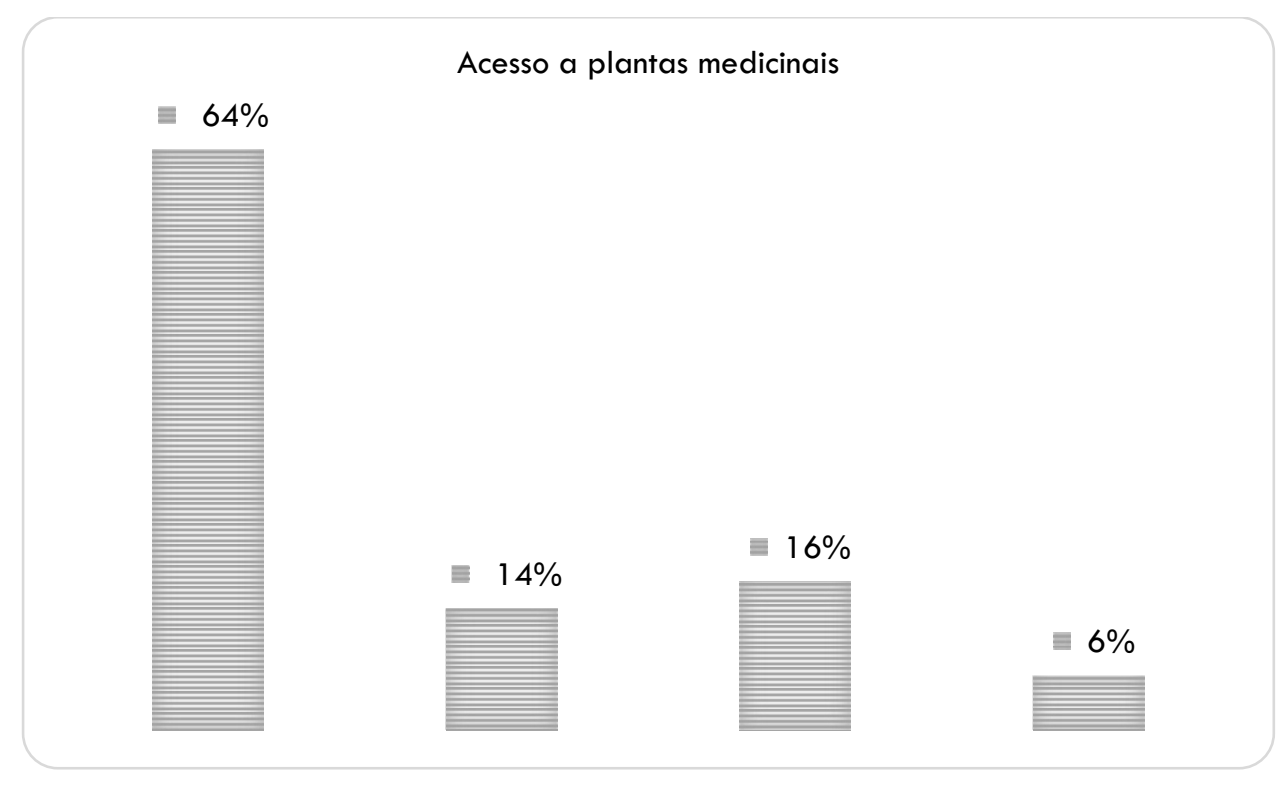

Gráfico 1 - Formas de acesso a produtos naturais 


\section{Egitania \\ $s$ c i e $\Omega$ c i a}

\section{DISCUSSÃO}

No nosso estudo maioritariamente os pais não recorrerem ao uso de produtos naturais como alternativa ou como terapêutica no tratamento das patologias da infância.

O recurso a estas substâncias é mais elevado nas gripes e constipações $(38,1 \%)$, tosse $(37,3 \%)$ e afeções respiratórias (22,9\%). Nas perturbações do sono e nas cólicas os valores percentuais de utilização assumem alguma expressividade estatística (20,3\%), para ambas as situações.

Nas afeções respiratórias o eucalipto recebe o maior número de respostas. A percentagem de 18,5\% de utilização demonstra, conforme bibliografia consultada (Jean \& Cyr, 2007), desconhecimento das implicações da inadequada utilização desta substância nesta afeção, pois ao aumentar a hiper-reatividade brônquica acresce o risco de alergia. Os diversos autores consultados (Cunha, Ribeiro \& Roque, 2009; Murgia \& Pagiotti, 2011; Loureiro, 2012) apontam o funcho e a sálvia (no nosso estudo apenas obteve 1 resposta) como mais adequadas. Também a hidratação intensa e o uso de infusões de mel e limão seriam mais benéficos nesta situação, podendo ainda ser utilizadas em idade inferior a dois anos, o que não acontece com o eucalipto que só está indicado a partir dos 6 anos (Loureiro, 2012).

Em situações de tosse a literatura (Loureiro, 2012) indica a cenoura, o mel ou o limão e foram estas as principais respostas obtidas14,9\% para o mel, 7,4\% para o limão e 9,1\% para a cenoura. Ora se existirem secreções é necessária hidratação intensa e estas substâncias ajudam no processo. $\mathrm{O}$ mel pode ainda controlar a inflamação da garganta. A grindélia, a hera, o alcaçuz, a ipecacuanha e a polígola são plantas indicadas na tosse (Cunha, Ribeiro \& Roque, 2009; Murgia \& Pagiotti, 2011), porém não foram referidas pelos nossos inquiridos.

No caso das afeções da orofaringe obteve-se uma resposta correta, a sálvia. O mel e o limão exercem um efeito emoliente e calmante na mucosa, mas não são as mais indicadas (Loureiro, 2012). Segundo a mesma autora par além da sálvia, o tomilho, a equinácea e a calêndula seriam mais indicadas, porém não foram referidas pelos nossos inquiridos o que pode indiciar algum desconhecimento sobre as indicações terapêuticas destas substâncias nas afeções pediátricas.

Relativamente às perturbações do sono constatámos que $13,5 \%$ dos pais referem recorrer à camomila e $2,4 \%$ à tília. A precaução acrescida em pediatria, nesta perturbação, estaria relacionada com a utilização de plantas como a passiflora, a verbena, erva-luísa, a tília, a cidreira e a valeriana em associação com alguns fármacos convencionais (Cunha, Ribeiro \& Roque, 2009; Murgia \& Pagiotti, 2011) pela possível interação farmacológica. O forte investimento por parte da indústria farmacêutica em produtos indutores do sono, baseados em produtos naturais, perspetiva uma resposta cada vez mais eficaz e ajustada a este problema.

Em situações de obstipação verificámos a utilização de xarope de maçãs reinetas $(5,9 \%)$ e de funcho $(2,5 \%)$. Esta utilização segundo a literatura consultada (Murgia \& Pagiotti, 2011) constitui-se um recurso terapêutico correto podendo nesta situação, como afirmam os mesmos 


\section{Egitania \\ $s$ c i e $\Omega$ c i a}

autores, recorrer-se de igual forma a emolientes e lubrificantes como o azeite, osmóticos como as ameixas, figos e uvas e a estimulantes de secreção e de motilidade como o sene e o óleo de rícino.

Nas cólicas, afeção frequente em pediatria, sobretudo em lactentes, a maior utilização recai sobre a cidreira $(5,7 \%)$ e o funcho $(4,1 \%)$, mas também sobre a camomila $(3,4 \%)$. As apresentações comerciais que contêm estas substâncias com propriedades calmantes (Loureiro, 2012), reconhecidas pelos profissionais de saúde e público geral, permitem admitir uma maior utilização destas substâncias. Porém os resultados obtidos nesta investigação revelam o contrário, ou seja, a sua reduzida utilização. Alguns autores (Murgia \& Pagiotti, 2011) referem um ainda elevado desconhecimento desta utilização terapêutica e remetem para a necessidade de aumentar as prescrições e aconselhamento destes produtos, por parte dos profissionais de saúde.

$\mathrm{Na}$ dermatite atópica obtivemos apenas uma resposta de utilização identificando a camomila como a substância utilizada. No entanto, de acordo com a literatura consultada (Cunha, Ribeiro \& Roque, 2009; Murgia \& Pagiotti, 2011; Loureiro, 2012), o aloés, a alteia, o alcaçuz e chá verde, seriam melhor opção. Quanto aos óleos, tendo em conta os autores referenciados, o de jojoba, o de borragem, o de hipericão e o de onagra seriam os mais indicados. No caso da dermatite irritativa da fralda as plantas medicinais mais indicadas seriam a alfazema e a propólis.

A elevada percentagem (64\%) de inquiridos que referem utilizar as plantas/produtos naturais por livre iniciativa revela, a nosso ver, um conhecimento empírico destas substâncias. Apenas $16 \%$ recorrem à sua utilização por indicação médica e $14 \%$ por aconselhamento farmacêutico. Estes resultados vão de encontro a estudos consultados (Jean \& Cyr, 2007; Miller, Hume, Harris, Jackson, Kanmaz, Cauffield \& Knell, 2000). Também Canário em 2001, refere a este respeito que os indivíduos que utilizam fitoterápicos geralmente não informam dessa utilização.

Num estudo (Welna, Hadsall \& Schommer, 2002) realizado com a finalidade de avaliar a utilização e o aconselhamento de produtos fitoterápicos, de 533 farmacêuticos entrevistados, $282(53 \%)$ assumiram o uso pessoal destes produtos. Os farmacêuticos que exercem a atividade profissional em farmácias comunitárias e residentes em regiões de maior interioridade são os que mais aconselham estes produtos. Quase todos os inquiridos (95\%) consideraram que as informações disponíveis sobre fitoterápicos não são "adequadas". Metade dos farmacêuticos (51\%) consideram os fitoterápicos seguros, mas apenas 19\% acredita na sua eficácia. Pouco mais da metade (56\%) referem recomendá-los aos seus utentes.

Lorenc num artigo de revisão tentou identificar o modelo, quer para profissionais de saúde, quer para progenitores, subjacente ao processo da tomada de decisão acerca da utilização destas substâncias (Lorenc, Ilan-Clarke, Robinson \& Blair, 2009). Concluiu que a compreensão do processo é crucial à tomada de decisão, promovendo uma decisão partilhada entre profissionais de saúde e pais. 


\section{Egitania \\ $\mathrm{s}$ c i e $\Omega$ c i a}

\section{CONCLUSÕES}

Os resultados obtidos na presente investigação dão-nos algumas orientações sobre a utilização de plantas/produtos naturais com fins terapêuticos usados na pediatria. Em número ainda reduzido, verificou-se a utilização destes produtos no tratamento de pequenas afeções das crianças e jovens da consulta de pediatria da Unidade Local de Saúde do Nordeste embora, em alguns casos, nem sempre com as indicações terapêuticas mais corretas.

Fica a necessidade de abordar de uma forma mais integrada esta problemática no seio dos profissionais de saúde, visando um conhecimento mais aprofundado destas substâncias para uma utilização ajustada a um quadro de prevenção ou tratamento de patologias desta faixa etária.

Apesar de a literatura apontar para progressos nesta área consideramos que a informação aos profissionais de saúde poderá conduzir a melhores resultados em termos de aconselhamento e utilização desta terapêutica. A atual aposta na investigação e no desenvolvimento de novas formulações, algumas derivações de moléculas já conhecidas pretendem, entre outros aspetos, dar resposta a problemas mais complexos, a resistência a antibióticos por exemplo.

Prevalece, ainda, alguma confusão entre medicamentos de síntese química e medicamentos naturais, nem sempre fácil de dissociar na população em geral e nos profissionais de saúde em particular.

A criação de observatórios, de grupos de pesquisa e de apoio à investigação por parte da indústria farmacêutica e de outras fontes de financiamento, levaria a uma utilização mais segura destas substâncias e provavelmente a um aumento da sua prescrição. A Universidade de Coimbra criou o primeiro Observatório de Interações Planta-Medicamento (OIPM) para conhecer e controlar este risco.

\section{BIBLIOGRAFIA}

Bhattaram V., Graefe U., Kohlert C., Veit M. \& Derendorf H. (2002). Pharmacokinetics and bioavailability OF HERBAL MEDICINAL PRODUCTS. PHYTOMEDICINE; 9:1-33.

Brunton L L, Chabner BA \& Knollmann BC (2012). As Bases Farmacológicas da Terapêutica (12ªd.). Porto ALEGRE: ARTMED/McGraw HiLl.

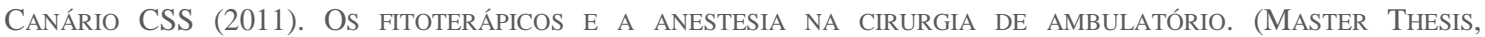
UNIVERSIDADE DA BEIRA INTERIOR). RETIRADO EM 12 DE SETEMBRO DE 2013 DE

HTTPS://UBIBLIORUM.UBI.PT/HANDLE/10400.6/915?LOCALE=EN

Ceolin T, Heck RM, Barbieri Rl, Schwartz E, Muniz RM \& Pillon CN. (2011). Plantas medicinais: TRANSMISSÃO DO CONHECIMENTO NAS FAMÍLIAS DE AGRICULTORES DE BASE ECOLÓGICA NO SUL DO RS. REVISTA ESCOLA ENFERMAGEM USP; 45: 47-54.

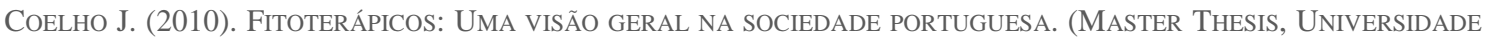
Fernando Pessoa). RetiRAdo EM 20 DE OUtUbro de 2013 DE 


\section{Eqitania \\ s c i e $\Omega$ c i a}

HTTP://BDIGITAL.UFP.PT/BITSTREAM/10284/1928/2/MONO_9764.PDF

Cunha AP \& Roque O. (2011). Plantas medicinais da FARMacopeia PORTUGUesa, CONSTituintes, CONTROLO, FARMACOLOGia E UTILIZAÇÃo (2a EDIÇÃo). LisboA: FundAÇÃo CALOUSTE GulbENKIAN.

Cunha AP, Ribeiro J \& Roque O. (2009). Plantas ARomáticas em Portugal, CARACTERIZAÇão e utilizaÇões (2a ED.). Lisboa: FundaÇão CAlouste GulbEnKian.

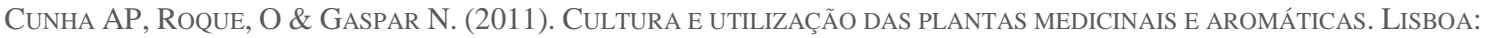
FundAÇÃO CALOUSTE GULBENKIAN.

Cunha AP, Silva A, Roque O \& Cunha E. (2011). Plantas e Produtos vegetais em cosmética e dermatologia ( $3^{a}$ ED.) Lisboa: FundaÇão CALOUSTE GULBENKIAN.

Cunha AP, Teixeira F, Silva A \& Roque O. (2010). Plantas na terapêutica, Farmacologia e ensaios Clínicos (2a ED.). Lisboa: FundaÇão CALOUSTE GulBEnKian.

Cunha AP. (2003). Aspectos Históricos Sobre Plantas Medicinais, Seus Constituintes Activos e Fitoterapia, Plantas e Produtos Vegetais em Fitoterapia ( $3^{a}$ ED.). Lisboa: Fundação Calouste Gulbenkian.

Delgado Sánchez O, Martínez lópez I, Crespí Monjo M \& Guerra Soler, G. (2008). Conciliación de la

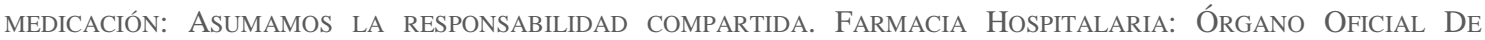
Expresión Científica De La Sociedad Española De Farmacia Hospitalaria: 32: 63-64.

AgÊNCIA Europeia do MEdicAmento (2013). RETIRAdo EM 05 DE OUtUbro de 2013 DE

HTTP://WWW.EMA.EUROPA.EU/HTMS/GENERAL/CONTACTS/HMPC/HMPC.HTML.

Fintelmann V \& Weiss RF. (2010). Manual de fitoterapia (11 ${ }^{a}$ ED.). Rio de Janeiro: Guanabara Koogan S.A.

Firmo W, Meneses V, Passos C, Dias C, Alves L, Dias I, Olea R. (2011). Historical context, popular use and SCIENTIFIC CONCEPTION ON MEDICINAL PLANTS. CAD. PESQ., SÃo Lú́s; 18: 90-95.

García E \& Solis I. (2007). MANUAl DE Fitoterapia. BARCELONA: ElSEVIER MASSON.

Gruenwald J, Brendler T, JANicKe C. (2007). PDR for herbal medicines (4ª Ed.). MontVale: Thomson.

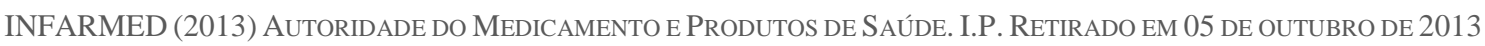
$\mathrm{DE}$

HTTP://WWW.INFARMED.PT/PORTAL/PAGE/PORTAL/INFARMED.

IZZO AA. (2005). HERB-DRUG INTERACTIONS: AN OVERVIEW OF THE CLINICAL EVIDENCE. FundAMENTAL \& CliniCAL PHARMACOLOGY; 19: 1-16.

JEAN D \& CYR C. (2007). USE OF COMPLEMENTARY AND ALTERNATIVE MEDICINE IN A GENERAL PEDIATRIC CLINIC. PEDIATRICS; 120:138-141.

KeMPER KJ, Vohra S\& WALLS R. (2008). THE USE OF COMPLEMENTARY AND ALTERNATIVE MEDiCINE IN PEDIATRICS. PEDIATRICS;122: 1374-1386.

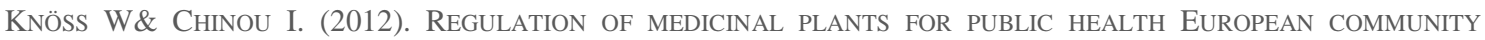
MONOGRAPHS ON HERBAL SUBSTANCES. PlANTA MEDICA; 78:1311-1316.

Lopes EM, Rodrigues Oliveira EA, Oliveira Lima L H, Feitosa Formiga LM \& Freitas RM. (2013). InTERAÇÕES

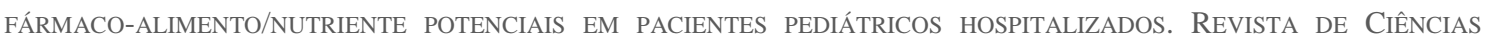
FARMACÊUTICAS BÁSICA E APLICADA; 34: 131-135. 


\section{Eqitania \\ s c i e $\Omega$ c i a}

Lorenc A, Ilan-Clarke Y, Robinson N \& Blair M. (2009). How parents Choose touse CAM: A systematic REVIEW OF THEORETICAL MODEls. BMC COMPLEMENTARy AND Alternative Medicine; 9: 9.

Loureiro S. (2012). Guia de RemÉdios naturais Para CrianÇAS (1ª ED.). Amadora: NASCENTE.

Martins AFG. (2010). Fitoterapia NA ABordagem do RisCo CARDiovascular: EFeItos do EXTRACTO DE ARROZ FERMENTADO POR MONASCUS PURPUREUS NO PERFIL LIPÍDICO. (MASTER THESIS, UNIVERSIDADE DA BEIRA INTERIOR). RETIRADO EM 11 DE NOVEMBRO 2013 DE

HTTPS://UBIBLIORUM.UBI.PT/HANDLE/10400.6/815

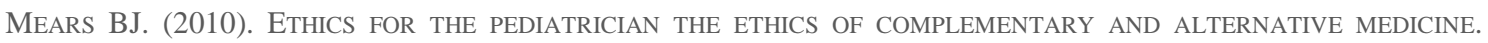
PEDiATRICs IN REVIEW; 31: 49-51.

Miller LG, Hume A, Harris IM, Jackson EA, Kanmaz TJ, Cauffield JS, Knell M. (2000). White Paper on herbal products. Pharmacotherapy: The Journal of Human Pharmacology and Drug Therapy; 20: 877-887.

Murgia V \& Pagiotti R. (2011). Problemas da CRiançA, AbORdagem integrada COM as Plantas medicinais. LISBOA: ABOCA.

Nascimento, Luís. (2013). Uso de Matrizes Naturais em Pediatria.(Master Thesis, Instituto Politécnico de BraganÇa e Universidade De SALAMANCA). RETIRAdo EM 12 JANEIRO DE 2014 DE

HTTPS://BIBLIOTECADIGITAL.IPB.PT/HANDLE/10198/9690

Pires, MCM da Silva. (2011). O QUE ACONTECE À MEDICAÇÃo CRÓNICO APÓS INTERNAMENTO HOSPITALAR? (MASTER Thesis, Universidade DA BEIRA INTERIOR). RETIRAdo EM 30 DE JANEIRO DE 2014 DE

HTTPS://UBITHESIS.UBI.PT/HANDLE/10400.6/996

RÄGo L \& SAntoso B. (2008). Drug REgulation: History, PRESENT and future. Drug Benefits and Risks: InTERnAtional TEXtbook of Clinical Pharmacology, Revised 2ND Edition, Edited by van BoXtel CJ, SANTOSO B, EDWARdS IR. IOS PRESS AND UPPSALA MONITORING CENTRE.

SChulz V, HANSEl R, \&Tyler VE.(2002). Fitoterapia RACIONAL: UM GUiA DE FITOTERAPIA PARA AS CIÊNCIAS DA SAÚDE. BARUERI: MANOLE.

Sibinga EM, Ottolini MC, Duggan AK\& Wilson MH. (2004). Parent Pediatrician communication about COMPLEMENTARY AND ALTERNATIVE MEDICINE USE FOR CHILDREN. CLINICAL PEDIATRICS; 43: 367-373.

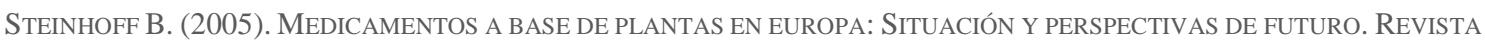
DE FITOTERAPIA; 5: 19-29.

Welna EM, Hadsall RS \& Schommer JC. (2002). Pharmacists' PERSONAl use, PROFESSiOnAl PRACTICE BeHAVIORS, AND PERCEPTIONS REGARDING HERBAL AND OTHER NATURAL PRODUCTS. JOURNAL OF THE AMERICAN PHARMACISTS ASSOCIATION: JAPHA; 43: 602-611.

Organização Mundial de Saúde (2001). Legal Status of Traditional Medicine and Complementary/Alternative Medicine: A Worldwide Review. Ginevra: WHO. 